\title{
Étude sur la microflore intestinale et sur les transformations biliaires chez des souris alimentées avec du yaourt ${ }^{(1)}$
}

\author{
par \\ N. PACINI*, A. FERRARI*, E. CANZI* \\ et B. BIANCHI-SALVADORI**
}

\section{INTRODUCTION}

Le yaourt est à même d'exercer tous ses effets positifs lorsque les bactéries qu'il contient réussissent à rester vivantes même après leur passage à travers le tractus gastro-intestinal.

Bianchi-Salvadori et al. $[1,2]$ ont démontré la présence de cellules de $L$. bulgaricus et de $S$. thermophilus dans les selles d'enfants et d'adultes, aussi bien après administration de ces bactéries dans le yaourt que sous forme de cellules lavées, pour éviter l'éventuelle action protectrice, exercée par la caséine. Ces résultats semblent être en désaccord avec les expériences menées in vitro par Lembke [3], visant à démontrer que les lactobacilles, et en particulier le L. bulgaricus, sont détruits en quelques secondes au contact de solutions à 0,47 p. 100 de $\mathrm{HCl}$. Pette [4] a formulé une hypothèse selon laquelle, dans l'organisme, l'acide chlorhydrique agirait dans des conditions complètement différentes de celles mentionnées ci-dessus, étant donné que, dans le suc gastrique, il se trouve en présence de protéines et de sels minéraux à action tampon.

Bianchi-Salvadori et Ferrari [5] ont du reste récemment démontré in vitro que des cellules de L. bulgaricus et de S. thermophilus en association survivent à l'action d'inhibition de l'acide désoxycholique, jusqu'à une concentration de 0,25 p. 100, qui est une valeur supérieure à la concentration normalement présente dans le cæcum de l'homme $(0,05-0,2$ p. 100$)$.

* Istituto di Microbiologia Agraria e Tecnica, Università di Milano.

** Centro Sperimentale del Latte, Milano.

(1) Cette étude a été exécutée avec la contribution du C.N.R. 
Chez les animaux supérieurs, l'activité de la microflore intestinale sur les acides biliaires exerce un rôle important sur la circulation entéropathique de ces composés et, par conséquent, sur leur métabolisme dans l'organisme.

Les principales activités exercées par les bactéries intestinales sur les acides biliaires sont représentées par la déconjugaison des acides conjugués et par la $7 \alpha$-désoxhydrilation des acides primaires libres.

Des études menées par différents auteurs sur la capacité déconjugante des lactobacilles intestinaux ont abouti à des résultats divers. Hill et Drasar [6], Aries et al. [7], et Drasar et Hill [8] ont constaté que des espèces de lactobacilles, isolés dans des selles humaines, étaient incapables de déconjuguer les acides taurocholique et glycocholique, alors que d'autres auteurs $[9,11]$ ont relevé cette capacité pour divers lactobacilles. A l'heure actuelle, l'on ne connaît encore aucun lactobacille capable d'effectuer la désoxhydrilation en $7 \alpha$ - des acides biliaires primaires, alors que cette capacité a été relevée en ce qui concerne le Streptococcus faecalis [6].

Au cours de recherches concernant l'influence de la diète sur l'activité de la microflore intestinale sur les acides biliaires, nous avons essayé de mettre en évidence les éventuelles variations, provoquées par l'administration de yaourt à des souris, sur les bactéries cæcales anaérobies à même d'hydrolyser les acides biliaires conjugués et de désoxhydriler l'acide cholique en $7 \alpha$ -

Dans la présente étude, nous avons également vérifié si l'on pouvait retrouver dans le cæcum de ces rongeurs les bactéries administrées avec le yaourt, après leur passage à travers le tractus gastrointestinal.

\section{MATERIEL ET METHODES}

\section{II.1. Animaux utilisés pour les expériences}

Les expériences ont été effectuées sur trente souris, souche Swisse Webster, pesant environ $25 \mathrm{~g}$. D'un premier lot de souris, à l'aide des tables des nombres randomisés [12], on a choisi les individus destinés à être utilisés dans les deux traitements. Les souris ont été mises dans deux cages, contenant chacune quinze souris, à une température de $25^{\circ} \mathrm{C}$.

\section{II.2. Traitement}

L'expérience a eu une durée totale de 7 semaines. Le régime normal a consisté en une alimentation ad libitum de $\mathrm{H}_{2} \mathrm{O}$ et de nourriture normale de la Maison Nossan (Milan-Italie). 
Après 1 semaine d'acclimatation et pendant $30 \mathrm{j}$ consécutifs, il a été administré également, aux souris appartenant à l'un des deux groupes, à l'aide d'une sonde gastrique, $1 \mathrm{ml}$ de yaourt deux fois par jour, avec un intervalle de $8 \mathrm{~h}$.

Après un jeûne de $18-20 \mathrm{~h}$, trois souris de chaque groupe ont été sacrifiées, dans l'ordre suivant : après 1 semaine d'acclimatation (heure 0 ), 15 et $30 \mathrm{j}$ après le début de l'administration de yaourt, $45 \mathrm{j}$ après, c'est-à-dire 2 semaines après la fin du traitement.

\section{II.3. Prélèvement des échantillons cæcaux et préparation de la suspension initiale}

Les souris sacrifiées ont été placées dans une cabine pour anaérobiose en plastique rigide (Tecnolabo, Milan, Italie), dans laquelle l'air a été remplacé par un mélange de gaz constitué par $\mathrm{N}_{2} / \mathrm{H}_{2} / \mathrm{CO}_{2}$ (85:10:5,v/v/v), stérilisés par filtration (FHLP 02500 Millipore) [13].

Sur trois souris, on a prélevé la portion centrale du cæcum, et les contenus ont été recueillis pour constituer un " pool » qui a été mis en suspension dans $18 \mathrm{ml}$ de solution désoxydante [14].

\section{II.4. Détermination des charges totales des bactéries anaérobies des contenus cæcaux}

Avec la suspension initiale, on a préparé deux séries de dilutions successives, en double, directement en bouillon de culture de Marcus et Talalay [15] (M.T.), précédemment réduit pendant 3 j en Gas Pak à $37^{\circ} \mathrm{C}$, pour la détermination des charges totales des bactéries anaérobies (première série) et des seuls anaérobies qui tolèrent l'oxygène (deuxième série).

On a opéré de deux façons différentes :

Première série : toutes les opérations (ouverture des animaux tués, prélèvement et dilution de l'échantillon cæcal, introduction des éprouvettes dans des récipients Gas Pak BBL pour l'incubation) ont été effectuées en cabine d'anaérobiose réalisant ainsi une chaîne continue d'anaérobiose rigoureuse.

Deuxième série : seuls l'ouverture des animaux tués, le prélèvement de l'échantillon cæcal et la préparation de la suspension initiale ont été effectués en cabine, alors que les opérations successives ont été faites en aérobiose ; tandis que l'incubation a été effectuée en Gas Pak.

Les récipients Gas Pak ont été incubés à $37^{\circ} \mathrm{C}$ pendant $7 \mathrm{j}$.

Le nombre total des bactéries a été déterminé par la technique du Most Probable Number (MPN), en utilisant deux éprouvettes pour chaque dilution. 
La charge totale des bactéries anaérobies tolérant l'oxygène a été vérifiée également en TGP [16] avec gélose, en opérant en double plaques, qui ont été mises en incubation en Gas Pak pendant $5 \mathrm{j}$, à $37^{\circ} \mathrm{C}$. Pour le comptage sur plaque, on a employé la méthode de Cavalli-Sforza [12].

Le poids sec des échantillons a été déterminé en plaçant $5 \mathrm{ml}$ de la suspension initiale dans un pèse-filtre ; on a séché en étuve à $110^{\circ} \mathrm{C}$, jusqu'à poids constant.

\section{II.5. Transformation des acides biliaires}

On a utilisé le bouillon de culture M.T., additionné des sels sodiques des acides biliaires. Les solutions des sels biliaires ont été stérilisées au moyen de membranes filtrantes Millipore (HAWF 01300).

Pour chaque acide biliaire, il a été préparé, en partant de la suspension initiale et au moyen de seringues à longue aiguille, deux doubles séries de dilutions, directement en bouillon précédemment réduit, dans les deux différentes conditions d'expérience précédemment décrites : soit dans la cabine pour anaérobiose soit en présence d'air.

L'incubation a été effectuée en Gas Pak.

\section{II.6. Extraction et chromatographies des acides biliaires}

Les cultures mixtes ont été acidifiées à $\mathrm{pH} 2,0$ avec $\mathrm{HCl} 5 \mathrm{~N}$ et extraites trois fois avec un mélange "chloroforme/méthanol " $(2: 1, \mathrm{v} / \mathrm{v})$; les extraits ont été chromatographiés sur plaques de gel de silice DC-Merk/0,25 mm d'épaisseur. Le mélange éluant était constitué par du benzène/acétate d'éthyle/acide acétique/ $\mathrm{H}_{2} \mathrm{O}$ (46:40:10:4, $\mathrm{v} / \mathrm{v} / \mathrm{v} / \mathrm{v})$. Les plaques ont été aspergées avec de l'acide sulfurique/ $\mathrm{H}_{2} \mathrm{O}$ $(1: 1, \mathrm{v} / \mathrm{v})$ et développées à $110^{\circ} \mathrm{C}$.

\section{II.7. Méthodes analytiques}

Les acides biliaires, séparés en éthers de méthyle sur colonne chromatographique ou en acides libres sur chromatographie en couche mince préparatoire (plaque de gel de silice de 0,5 $\mathrm{mm}$ d'épaisseur, DC Merk) ont été confirmés par la spectrométrie de masse à infrarouges.

On a utilisé un spectrophotomètre infracord (Perkin Elmer Mtd), pour obtenir les spectres infrarouges (en Nujol); les spectres de masse ont été obtenus avec un spectromètre LKB modèle 9000.

\section{II.8. Produits employés}

L'acide $3 \alpha, 7 \alpha, 12 \alpha$-trihydroxy-5 $\beta$-cholanique a été fourni par N.B.C. Cleveland Ohio U.S.A. ; l'acide $3 \alpha, 12 \alpha$-dihydroxy-5 $\beta$-cholanique par 


\section{TABLEAU 1}

Nombre (MPN) des bactéries anaérobies et des bactéries « oxygène - tolérantes " dans le contenu cæcal de souris traitées ou non au yaourt

(n bactéries/g de poids sec)

\begin{tabular}{|c|c|c|c|c|}
\hline \multirow{2}{*}{$\begin{array}{l}\text { Temps } \\
\text { en jours }\end{array}$} & \multicolumn{2}{|c|}{ Bactéries anaérobies } & \multicolumn{2}{|c|}{ Bactéries oxygène - tolérantes } \\
\hline & non traitées & traitées & non traitées & traitées \\
\hline 0 & $5,4 \quad 10^{10}$ & & $5,4 \quad 10^{8}$ & \\
\hline 15 & $7,1 \quad 10^{10}$ & $6,3 \quad 1010$ & $7,1 \quad 10^{8}$ & $6,3 \quad 10^{9}$ \\
\hline 30 & $1,5 \quad 10^{10}$ & $5,9 \quad 10^{9}$ & $1,5 \quad 1010$ & $5,9 \quad 10^{9}$ \\
\hline 45 & $6,2 \quad 10^{10}$ & $6,5 \quad 1010$ & $6,2 \quad 10^{9}$ & $6,5 \quad 10^{9}$ \\
\hline 0,01 & & & & \\
\hline
\end{tabular}

TABLEAU 2

Nombre total des bactéries anaérobies oxygène - tolérantes dans le contenu cæcal de souris traitées ou non au yaourt (n bactéries/g de poids sec)

\begin{tabular}{c|c|c}
\hline Temps en jours & Non traitées $(\overline{\mathrm{x}} \pm \sigma)$ & Traitées $(\overline{\mathrm{x}} \pm \sigma)$ \\
\hline 0 & $7,3 \pm 0,3.10^{8}$ & \\
\cline { 2 - 2 } & $5,4 \pm 0,4.10^{9}$ & $5.10^{9}$ \\
30 & $1,4 \pm 0,4.10^{10}$ & $5,8 \pm 0,3.10^{9}$ \\
45 & $3,8 \pm 0,2.10^{9}$ & $4,7 \pm 0,2.10^{9}$ \\
& & \\
\hline
\end{tabular}

$\overline{\mathrm{x}}=$ valeur moyenne $; \sigma=$ écart-type.

Applied Science Laboratories Inc., State College, U.S.A. ; l'acide $3 \alpha$, $12 \alpha$-dihydroxy-7-oxo-5 $\beta$-cholanique a été préparé comme décrit par Fiesher et Rajiagopalan [17] ; l'acide 5 $3-3 \alpha, 7 \alpha, 12 \alpha$-trihydroxycholanique-N (2 sulféothyle) amide, par Serva Feinbiochemica, Heidelberg. 


\section{RESULTATS}

\section{III.1. Charge totale des bactéries anaérobies}

Après le traitement des souris au yaourt, dans les laps de temps mentionnés, il a été procédé à l'évaluation des charges totales des bactéries anaérobies. Comme le démontrent les données du tableau 1 chez les souris traitées aux différents moments, le MPN des bactéries anaérobies est le même que celui des souris non traitées. Le comptage sur plaque des bactéries oxygène-tolérants ont confirmé les résultats obtenus dans le liquide (tab. 2). De plus (tab. 1) les bactéries ne tolérant pas l'oxygène sont de 10 à 100 fois plus nombreuses que celles qui tolèrent l'oxygène $(P \leqslant 0,01)$.

\section{III.2. Déconjugaison de l'acide taurocholique}

Après $5 \mathrm{j}$ d'incubation en bouillon M.T., à $37^{\circ} \mathrm{C}$, additionné de taurocholate de sodium à 0,02 p. 100, les extraits des cultures ont été chromatographiés en couche mince. L'activité déconjugante à été mise en évidence par la disparition de l'acide cholique $\left(R_{\ell} 0,30\right)$; on a pu également relever la présence d'autres métabolites, attribuables à d'éventuels produits de transformation, par suite de la séparation de ce composé de la taurine.

Les résultats concernant la déconjugaison de l'acide taurocholique, obtenus par la méthode du MPN par rapport au poids sec, sont représentés dans la figure 1 , en même temps que les résultats concernant les charges microbiennes des mêmes cultures mixtes.

Les résultats mettent en évidence le fait que le nombre des bactéries cæcales, capables d'opérer la déconjugaison de l'acide taurocholique, se présente dans la même proportion chez les souris traitées au yaourt et chez celles non traitées. De plus, il a été relevé une activité déconjugante dans toutes les dilutions, dans lesquelles il y a eu croissance. Il s'ensuit donc que, dans les deux conditions d'expérience adoptées, la croissance et, par conséquent, l'activité déconjugante a été de 1 à 2 fois plus forte dans les conditions d'anaérobiose continue.

\section{III.3. 7 andésoxhydrilation de l'acide cholique}

Après $5 \mathrm{j}$ d'incubation à $37^{\circ} \mathrm{C}$ en bouillon M.T. additionné de cholate de sodium à 0,01 p. 100 , les extraits des cultures ont été chromatographiés en couche mince.

L'activité $7 \alpha$-désoxhydrilante a été mise en évidence par l'apparition de l'acide désoxycholique $\left(R_{\mathrm{r}} 0,63\right)$.

Pendant toute la durée de l'expérience, il n'a été relevé aucune différence substantielle dans la croissance et dans l'activité de transformation dans les contenus cæcaux de l'un ou l'autre groupe de souris. 


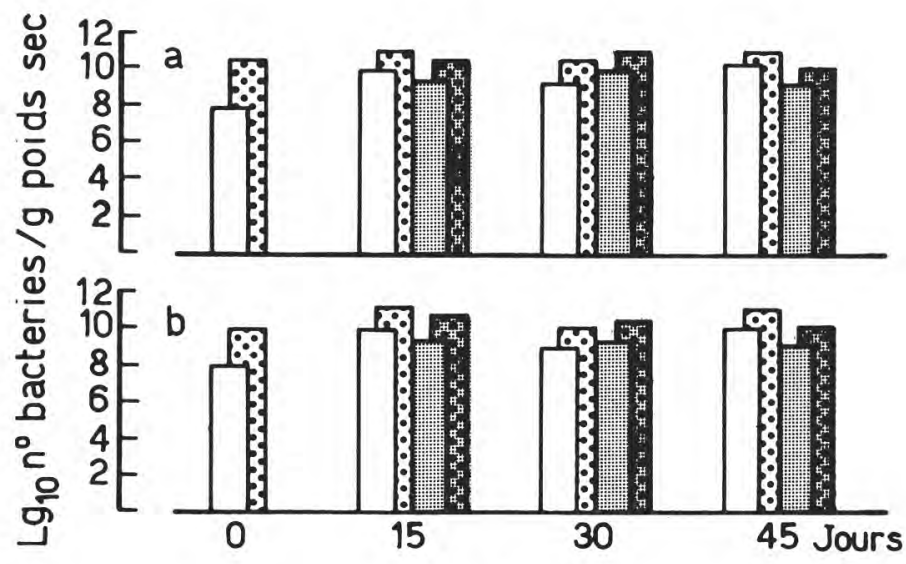

fig. 1

Charges totales (a) et bactéries déconjugantes de l'acide taurocholique (b) du contenu cæcal de souris

Bactéries oxygène-non tolérantes du contenu cæcal de souris non traitées rantes du contenu cæcal de souris non traitées $\square$ et de souris traitées

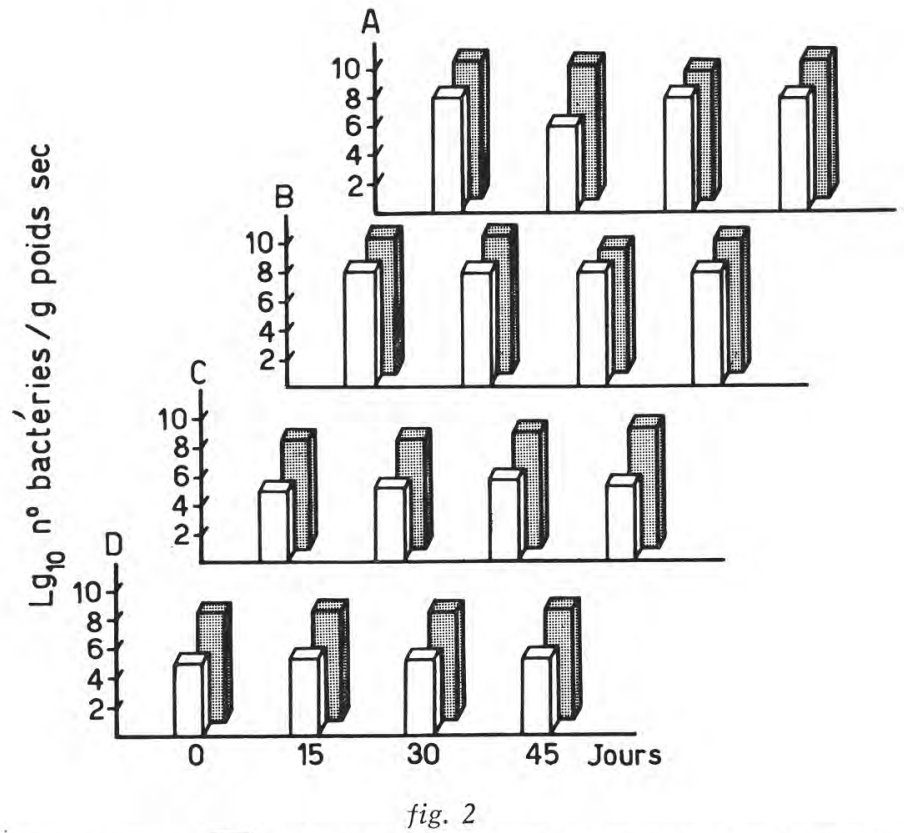

Charges totales 3 et bactéries $7 \alpha$-désoxhydrilantes 1'acide cholique $\square$ du contenu cæcal de souris non traitées (A et C) et traitées (B et D) au yaourt. Bactéries ne tolérant pas l'oxygène (A et B) ; bactéries oxygène-tolérantes (C et $\mathrm{D}$ ). 
Les résultats concernant la 7 $\alpha$-désoxhydrilation de l'acide cholique, obtenus avec la méthode du MPN par rapport au poids sec, sont représentés dans la figure 2 , en même temps que ceux concernant les charges microbiennes des mêmes cultures mixtes.

Dans les conditions d'anaérobiose rigoureuse, il s'est avéré que la transformation se produisait à des dilutions plus fortes, dans un ordre de grandeur d'environ 3 fois plus, bien que le degré de croissance des cultures ne diffère que de 1-2 ordre de grandeur.

L'analyse chromatographique des extraits des cultures a mis en évidence la présence d'une tache avec $R_{\mathrm{f}} \quad 0,45$ attribuable à l'acide 7-chétodésoxycholique. Cette tache était également relevable dans les cultures provenant des dernières dilutions, incapables de 7 $\alpha$-désoxhydriler l'acide cholique. La capacité d'oxyder l'oxhydrile en 7 $\alpha$ - de l'acide cholique, de la part des bactéries des contenus cæcaux analysés, est donc plus fréquente que la capacité $7 \alpha$-désoxhydrilante.

\section{III.4. Recherche des bactéries du yaourt dans les contenus cæcaux}

Aux fins de vérifier l'éventuelle présence de bactéries du yaourt, en partant de plaques préparées au $15^{\mathrm{e}}$, au $30^{\mathrm{e}}$ et au $45^{\mathrm{e}} \mathrm{j}$, il a été effectué de nombreux isolements. Ce n'est qu'au $15^{\text {e }}$ et au $30^{\text {e }} \mathrm{j}$ que l'on a pu isoler des bactéries «Gram positives », qui présentaient les caractéristiques morphologiques, culturelles et biochimiques du $L$. bulgaricus. L'identification a été effectuée au moyen de l'Api System 50 Lactobacillus. L. bulgaricus a été décelé à raison de $10^{6} / \mathrm{g}$ de poids $\mathrm{sec}$ de contenu cæcal.

$\mathrm{Au} 45^{\mathrm{e}}$ jour, c'est-à-dire 2 semaines après la fin du traitement, cette forme microbienne s'est avérée introuvable.

\section{CONCLUSION}

La présence de L. bulgaricus dans le contenu cæcal de la souris, mise en évidence pendant le traitement au yaourt, a permis de constater que ces formes microbiennes, même chez cet animal, sont en mesure de franchir la barrière gastro-intestinale.

De plus, l'administration de yaourt n'a eu aucune influence sur la microflore anaérobie cæcale, qui a conservé sa capacité normale d'hydrolyser l'acide taurocholique.

Cette capacité s'est révélée très floue en ce qui concerne aussi bien les bactéries anaérobies tolérant l'oxygène que celles qui ne tolèrent pas l'oxygène. Cela a été démontré par le fait que le nombre des bactéries actives pour cette transformation s'est révélé être du même ordre de grandeur que celui de la charge totale des bactéries anaérobies.

D'ailleurs, divers auteurs ont signalé, chez de nombreuses formes microbiennes, cette capacité de déconjuguer les acides biliaires [8]. 
En ce qui concerne également la capacité de désoxhydriler l'acide cholique en $7 \alpha$, aucune variation n'a pu être décelée, suite au traitement au yaourt. Il semble toutefois que les bactéries ne tolérant pas l'oxygène soient les principales responsables de cette transformation microbienne, qui n'est pas beaucoup diffuse entre eux [18].

En ce qui concerne la capacité de transformer l'acide cholique en acide 7-chétodéoxycholique, on a pu relever qu'il s'agit là d'une propriété courante, même chez les bactéries qui tolèrent l'oxygène.

\section{R és u m é}

A la lumière des récentes recherches concernant l'influence des régimes sur la microflore intestinale, nous avons essayé d'analyser les éventuelles variations, produites par l'administration de yaourt à des souris, sur l'activité des bactéries anaérobies cæcales capables d'hydrolyser les acides biliaires conjugués et de désoxhydriler l'acide cholique en $7 \alpha$. Nous avons adopté des techniques qui exigent la mise en place d'une chaîne d'anaérobiose, depuis le prélèvement du contenu cæcal jusqu'à l'incubation des cultures, de manière à permettre le développement des bactéries ne tolérant pas l'oxygène, normalement présentes en grand nombre dans l'intestin des mammifères. Le traitement au yaourt n'a eu aucune influence, ni sur le nombre d'anaérobies ni sur l'activité de ces bactéries sur les acides biliaires ; par ailleurs, les bactéries ne tolérant pas l'oxygène se sont révélées les principales responsables de l'activité déconjugante sur l'acide taurocholique et $7 \alpha$-désoxhydrilante sur l'acide cholique. Nous avons pu également constater que L. bulgaricus, administré avec le yaourt, a été retrouvé vivant dans le caecum des rongeurs, après son passage à travers le tractus gastro-intestinal.

\section{S u m m a ry}

Taking in to account the recent researches on the influence of different diets on the intestinal microflora, we have tried to evaluate the possible effects, induced by feeding mice with yogurt, on the activity of anaerobic cæcal micro-organisms capable of hydrolizing conjugated bile acids and $7 \alpha$-dehydroxylating cholic acid. Using techniques which require an anaerobic chain from the sampling of the cæcal content to the incubation of the cultures we have obtained the development of $\mathrm{O}_{2}$ non tolerant anaerobic micro-organisms which are normally present in large numbers in the mammalian intestine. The treatment with yogurt did not show any effect nor on the number of the anaerobic micro-organisms nor on their activity on bile acids. The anaerobic oxygen non tolerant micro-organisms resulted the mainly responsible for the deconjugation of taurocholic acid and $7 \alpha$-dehydroxilation of cholic acid. Furthermore L. bulgaricus, supported by yogurt, has been 
found in a viable state in the cæcum of mice after having passed through the gastro-intestinal tract.

Reçu pour publication en août 1979.

\section{Bibliographie}

[1] Bianchi-Salvadori (B.), Brughera (F.), Salvadori (P.) (1967). - Studio sulle variazioni coprocolturali nel lattante, in rapporto alla somministrazione di yogurt. Min. Diet., 7, 176-180.

[2] Salvadori (P.), Bianchi-Salvadori (B.) (1973). - Studio sulle variazioni coprocolturali nell'uomo in rapporto alla somministrazione di yogurt. Min. Diet., 13, 8-12.

[3] LembKe (A.) (1964). - Latti fermentati ed abitudini alimentari. Rapport $n^{\circ} 3$, Bull. Ann. F.I.L.I.D.F., Les laits fermentés, 22-34.

[4] Pette (J. W.) (1964). - Caractéristiques des souches communément utilisées pour la préparation des laits fermentés les plus importants. Rapport $n^{\circ} 7$, Bull. Ann. F.I.L.-I.D.F., Les laits fermentés, 78-87.

[5] Bianchi-Salvadori (B.), Ferrari (A.) (1978), - Azione degli acidi biliari sui fermenti lattici dello yogurt. It Latte, 3 (4), 249-255.

[6] Hill (M. J.), Drasar (B. S.) (1968). - Degradation of bile salts by human intestinal bacteria. Gut, 9, $22-27$.

[7] Aries (V.), Crowther (J. S.), Drasar (B. S.), Hill (M. J.) (1969). - Degradation of bile salts by human intestinal bacteria. Gut, 10, 575-576.

[8] Drasar (B. S.), Hill (M. J.) (1974). - Human intestinal flora. Academic Press, London, New York, San-Francisco.

[9] Midtvedt (T.), Norman (A.) (1967). - Bile acid transformation by microbial strains belonging to genera found in intestinal contents, Acta Path. Microbiol. Scandinav., 71, 629-638.

[10] Floch (M. H.), Binder (H. J.), Filburn (B.), Gershengoren (W.) (1972). The effect of bile acids on intestinal microflora. J. Clin. Nutr., 25, 1418-1426.

[11] Gilliland (S. E.), Speck (M. L.) (1977). - Deconjugation of Bile Acids by Intestinal Lactobacilli. Appl. Environ. Microbiol., 33, 15-18.

[12] Cavalli-Sforza (L.) (1966). - Analisi statistica per medici e biologi ed analisi del dosaggio biologico. Boringhieri, Torino, Italia.

[13] Fesce (A.), Ceccarelli (A.), Poli (G.), Balsari (A.), Giovannini (R) (1977). Metodo per l'isolamento e lo studio di batteri anaerobi ossigeno-intolleranti. Arch. Vet. Ital., 28, 1-9.

[14] Ferrari (A.), Pactni (N.), Ceccarelli (A.) (1975). - Attività dei microrganismi cecali ossigeno non tolleranti nella $7 \alpha$-deossidrilazione in vitro dell'acido colico. Ann. Microbiol., 25, 57-61.

[15] Marcus (P.), Talalay (P.) (1956). - Induction and purification of $\alpha$ and $\beta$-idrossisteroid dehydrogenases. J. Biol. Chem., 218, 661-674.

[16] Scardovi (V.), Trovatelli (L. D.), Zani (G.), Crociani (F.), Matteuzzi (D.) (1971). - Deoxyribonucleic Acid Homology Relationships Among Species of the Genus Bifidobacterium. Int. J. of Syst. Bacteriol., 21, 276-294.

[17] Fiesher (L. F.), Rajiagopalan (S.), - Selective oxidation with N-Bromosuccinimide. I. Cholic acid. J. Amer. Chem. Soc., 71, 3935-3938.

[18] Hayakawa (S.) (1973). - Microbiological Transformation of Bile Acids. Adv. Lipid Res., 2, 143-192. 